\title{
Exact evaluation of Equal-Gain Diversity in the presence of Nakagami fading
}

\author{
G. K. Karagiannidis and S. A. Kotsopoulos
}

\begin{abstract}
A closed formulation for the evaluation of the error probability of Lbranches Equal-Gain Combiners over Nakagami fading channels and additive Gaussian noise, is presented. Using the definition and the properties of the characteristic function of a sum of random variables, the error performance is evaluated directly for coherent detection and several modulation techniques (BPSK, BFSK).
\end{abstract}

Introduction: Diversity provides a powerful technique for combating fading in mobile communications systems. Equal-Gain Combining (EGC) appears to be an attractive diversity tool due to its easy implementation and its improved error performance, which is comparable to the optimal Maximal Ratio Combining (MRC) [1][2]. Several effective techniques have been proposed for the evaluation of the error probability (ERRP) for EGC over Nakagami fading channels. Beaulieu and Dayya in [3] have devised a direct evaluation of the ERRP through the determination of the probability density function (pdf) of the sum of $L$ Nakagami interferers. This is an important result in literature since it was the first time where such a technique can be used in the general case of L-order EGC diversity. However, the devised closed forms need the evaluation of two kinds of the complicated confluent hypergeometric function. Later, other methods are proposed for the evaluation of the ERRP for M-ary QAM [4][5]. Recently, the authors in [6] proposed a simpler formulation for the evaluation of the EGC ERRP over Rayleigh fading channels. This approach will be generalized in the present letter for the case of Nakagami fading environment with arbitrary values for the modeling parameters. The ERRP in EGC diversity systems with additive uncorrelated white noise is calculated directly using the definition and the properties of the CHF of the sum of random variables. A final closed formula, which contains $L$ summations for L-branch diversity, is derived. This formula is very simple, can be evaluated numerically using Hermite integration technique and it does not contain any complicated calculations (e.g confluent hypergeometric functions or infinite series) as previous techniques.

System model and error analysis: In an ECG system with coherent detection the signals received in each branch are co-phased, summed and coherently demodulated [1]. The decision variable $U$ for coherent BPSK can be formulated as 


$$
U= \pm \sum_{k=1}^{L} r_{k}+\sum_{k=1}^{L} w_{k}
$$

where $r_{k}$ is the output signal amplitude at the $k^{\text {th }}$ branch, which remains constant within symbol duration but changes from symbol to symbol. The first term in (1) assumes the positive sign if the transmitted symbol is one and assumes the negative sign if it is zero. The $r_{k}$ follows Nakagami m-distribution with pdf given by

$$
p_{r_{k}}(t)=\frac{2}{\Gamma(m)} \cdot\left(\frac{m}{\Omega_{k}}\right)^{m} \cdot t^{2 m-1} \cdot \exp \left(-\frac{m}{\Omega_{k}} \cdot t^{2}\right)
$$

whereas $\Gamma(x)$ is the Gamma function, $\Omega_{\mathrm{k}}$ represents the average signal $\mathrm{p}$ ower at $\mathrm{k}$ branch and $\mathrm{m}$ represents the inverse normalised variance $\mathrm{r}^{2}$. Acting.as a shape factor, $m$ is an arbitrary fading severity parameter and can take values from 0.5 through infinity. Setting the parameter $m$ to unity yields a Rayleigh pdf since a value of 0.5 yields a half- Gaussian pdf. Values of unity or less describe deep fading, while values much greater than unity describe shallow (Rician) fading [2]. Here it is assumed that the $\mathrm{m}$ parameter is the same for all branches, which is also true in real applications. The parameter $w_{k}$ in (1) represents the complex Gaussian noise at the $k^{\text {th }}$ branch with zero mean and variance $\mathrm{N}_{\mathrm{k}} / 2$. Let $\Phi_{U}(s), \Phi_{r_{k}}(s)$ and $\Phi_{w_{k}}(s)$ be the CHF of the parameter $\mathrm{U}, \mathrm{r}_{\mathrm{k}}$ and $w_{k}$ correspondingly. Assuming the positive sign in equation (1) and due to the independence between desired signals and noise in each branch, $\Phi_{U}(s)$ can be written as

$$
\Phi_{U}(s)=\prod_{i=1}^{L} \Phi_{w_{k}}(s) \cdot \prod_{k=1}^{L} \int_{0}^{\infty} \exp \left(j s x_{k}\right) \cdot p_{r_{k}}\left(x_{k}\right) d x_{k}
$$

The average bit energy to average noise density ratio (SNR) at the $k^{\text {th }}$ branch is defined here as

$$
\rho_{k}=\frac{\Omega_{k}}{\eta_{L} / L}
$$

with $\eta_{L}$ being the total power of the Gaussian noise (in all branches).

The ERRP for coherent BPSK detection is defined as [1]

$$
P_{e}=\operatorname{Pr} o b(U<0)=\int_{-\infty}^{0} p_{U}(\tau) d \tau=\frac{1}{2 \pi} \cdot \int_{-\infty-\infty}^{0} \int^{\infty} \Phi_{U}(s) \cdot \exp (-j s \tau) d s d \tau
$$

where $\tau$ is another auxiliary variable. After the transformation $x_{k}=\sqrt{\frac{t_{k} \cdot \Omega_{k}}{m}}$ and taking into account the fact that by definition

$$
\int_{-\infty}^{\infty} \Phi \Phi_{\text {NORM }\left(0, \frac{\eta_{L}}{2}\right)}(s) \cdot \exp \left[-j s\left(\tau+\sum_{k=1}^{L}-\sqrt{\frac{t_{k} \cdot \Omega_{k}}{m}}\right)\right] d s=2 \cdot \pi \cdot p_{\text {NORM }\left(0, \frac{\eta_{L}}{2}\right)}\left(\tau+\sum_{k=1}^{L}-\sqrt{\frac{t_{k} \cdot \Omega_{k}}{m}}\right)
$$

and following the same mathematical analysis as in [6] the ERRP can be written as

$$
P_{e}=\frac{1}{[\Gamma(m)]^{L}} \int_{0}^{\infty} \cdot \int_{0}^{\infty} P_{\text {NORM }(0,1)}\left(-\sum_{k=1}^{L} \sqrt{\frac{2 \cdot \rho_{k} \cdot t_{k}}{L \cdot m}} \cdot r_{k}\right) \cdot \prod_{k=1}^{L} t_{k}{ }^{m-1} \cdot d t_{1} \cdot \cdot d t_{L}
$$


In equation (6) $\Phi_{N O R M}\left(0, \frac{\eta_{L}}{2}\right)$ and $p_{N O R M}\left(0, \frac{\eta_{L}}{2}\right)$ are the CHF and the pdf of a Normal distribution with mean zero and variance $\eta_{L} / 2$ since in equation (7) $P_{\operatorname{NORM}(0,1)}(x)$ is the well-known standard normal cumulative distribution function (cdf). Equation (7) involves $L$ integrals for $L$ diversity branches and its second part can be calculated numerically with high desired accuracy using the Hermite numerical integration method [7]. Hence, the final closed form for the calculation of ERRP in a BPSK L-order EGC can be written as

$$
P_{e}=\frac{1}{[\Gamma(m)]^{L}} \cdot \sum_{i=1}^{v} \alpha_{i} \sum_{j=1}^{v} \alpha_{j} \cdot \sum_{n=1}^{v} \alpha_{n} \cdot F_{N O R M(0,1)}\left(-\sum_{k=i, j, \ldots, n} \sqrt{\frac{2 \rho_{k} \cdot z_{k}}{L \cdot m}}\right) \cdot \prod_{k=i, j, \ldots, n} z_{k}{ }^{m-1}
$$

with $\alpha, z, 2 v$ are the weight factors, the abscissas and the order of the Hermite numerical integration method. Because the integrals are defined over the positive half axes only the positive values of abscissas are used. In the case of coherent BFSK the noise variance is double than the corresponding in BPSK. Hence, the proposed formulation is also valid when $\rho_{k}$ is replaced by $\rho_{k} / 2$ in equation (8). Moreover, the new proposed approach can be also used perfectly for non-coherent DPSK detection. Analysis and the corresponding results of this case are not presented, due to the limited size of the present letter. It must be noted here that, in the case of perfect coherent detection and no co-channel interference, the ERRP performance of BPSK is identical to that of 4PSK [3]. As far as the computation time is concerned, when the order of diversity is smaller or equal than three the proposed formulation is simpler and faster than the corresponding in [3] although it presents a disadvantage of lengthy computation time when the order is greater than three. This disadvantage is counterbalanced with the offered simplicity and accuracy since it needs the calculation of well-known functions avoiding the evaluation of complicate functions such as the two types of confluent hypergeometric. The calculation of such functions - as it is also referred in [5, Appendix B]- presents serious time overflow problems under some circumstances.

Numerical results: First, we assume an EGC coherent BPSK system with the same signal levels among the branches (i.e $\rho_{1}=\rho_{2}=. . \rho_{\mathrm{k}}$ ). If it is not true, better performance may be obtained by adjusting the gains to give equal power in all branches [3]. However, equation (8) can be also used for the case of gain unbalancing between the branches. The ERRP at a reference branch is calculated for several values of the fading parameter $\mathrm{m}$ and order of diversity and the results are depicted in Figure 1. In Figure 2 the ERRP is related to the $m$ parameter for several values of SIR and for $L=2$ and $L=3$. We note here that the error performance increases for higher values of the Nakagami $\mathrm{m}$ parameter. A slight change in $\mathrm{m}$ leads to a significant change in ERRP, especially for large SNR. This happens because an increase of the $\mathrm{m}$ parameter means that the desired signal does not suffer from severe fading, which degrades the error performance. We also observe that higher order of diversity leads to an improvement to the error performance.

Conclusions: A novel simple approach for the evaluation of error performance in L-order equal-gain diversity over Nakagami fading channels, is presented. The obtained closed formulation can be easily used to demonstrate 
the error performance helping the designers to make decisions on crucial system parameters by taking into account the Quality of Service (Qos) demands.

\section{References}

1. PROAKIS, J.: Digital Communications, $2^{\text {nd }}$ Edition, McGeaw-Hill.

2. STEELE, R., and HANZO, L.: Mobile Radio Communications, Wiley Publications, 1999.

3. BEAULIEU, N., and ABU-DAYYA, A.: 'Analysis of equal gain diversity on Nakagami fading channels', IEEE Transactions on Communications, February 1991, Vol. 39, No. 2, pp. 225-234.

4. ALOUINI, M., and GOLDSMITH, A.: 'A unified approach for calculating error rates of linearly modulated signals over generalized fading channels', in Proc. ICC'98, Atlanta, GA, June 1998, pp. 459-464.

5. ANNAMALAI, A., TELLAMBURA, C., and BHARGAVA, V.: Exact evaluation of maximal-ratio and equal-gain diversity receivers for $\mathrm{M}$-ary QAM on Nakagami fading channels', IEEE Transactions on Communications, Sep. 1999, Vol. 47, No. 9, pp. 1335-1344.

6. KOTSOPOULOS, S., and KARAGIANNIDIS, G.: "Error performance for equal-gain combiners over Rayleigh fading channels', IEE Electronics Letters, Vol. 36, Issue 10.

7. GRADSHTEYN, S., and I. M. RYZHIK, I.: Table of Integrals, Series and Products. New York: Academic Press, 1980.

\section{Authors' affiliations:}

G. K. Karagiannidis and S. A. Kotsopoulos (Department of Electrical \& Computer Engineering, Wireless Telecomm. Lab., University of Patras, Patras, 26110, GREECE) 


\section{Figure captions:}

Fig. 1 Error probability versus SNR at a reference branch for several order of diversity and values of the Nakagami $\mathrm{m}$ parameter

$\begin{array}{ll}\longrightarrow & \mathrm{m}=1.5 \\ \cdots & \mathrm{m}=4.5 \\ \mathrm{O} & \mathrm{L}=2 \\ \mathrm{x} & \mathrm{L}=3\end{array}$

Fig. 2 Error probability versus the Nakagami m parameter for several values of SNR at a reference and diversity orders

\begin{tabular}{|c|c|}
\hline ----- & $\begin{array}{l}L=2 \\
L=3\end{array}$ \\
\hline O & $S N R=0 \mathrm{~dB}$ \\
\hline$x$ & $S N R=5 \mathrm{~dB}$ \\
\hline$\square$ & $\mathrm{SNR}=10 \mathrm{~dB}$ \\
\hline
\end{tabular}


Figure $1 \mathrm{G}$. Karagiannidis and S. Kotsopoulos

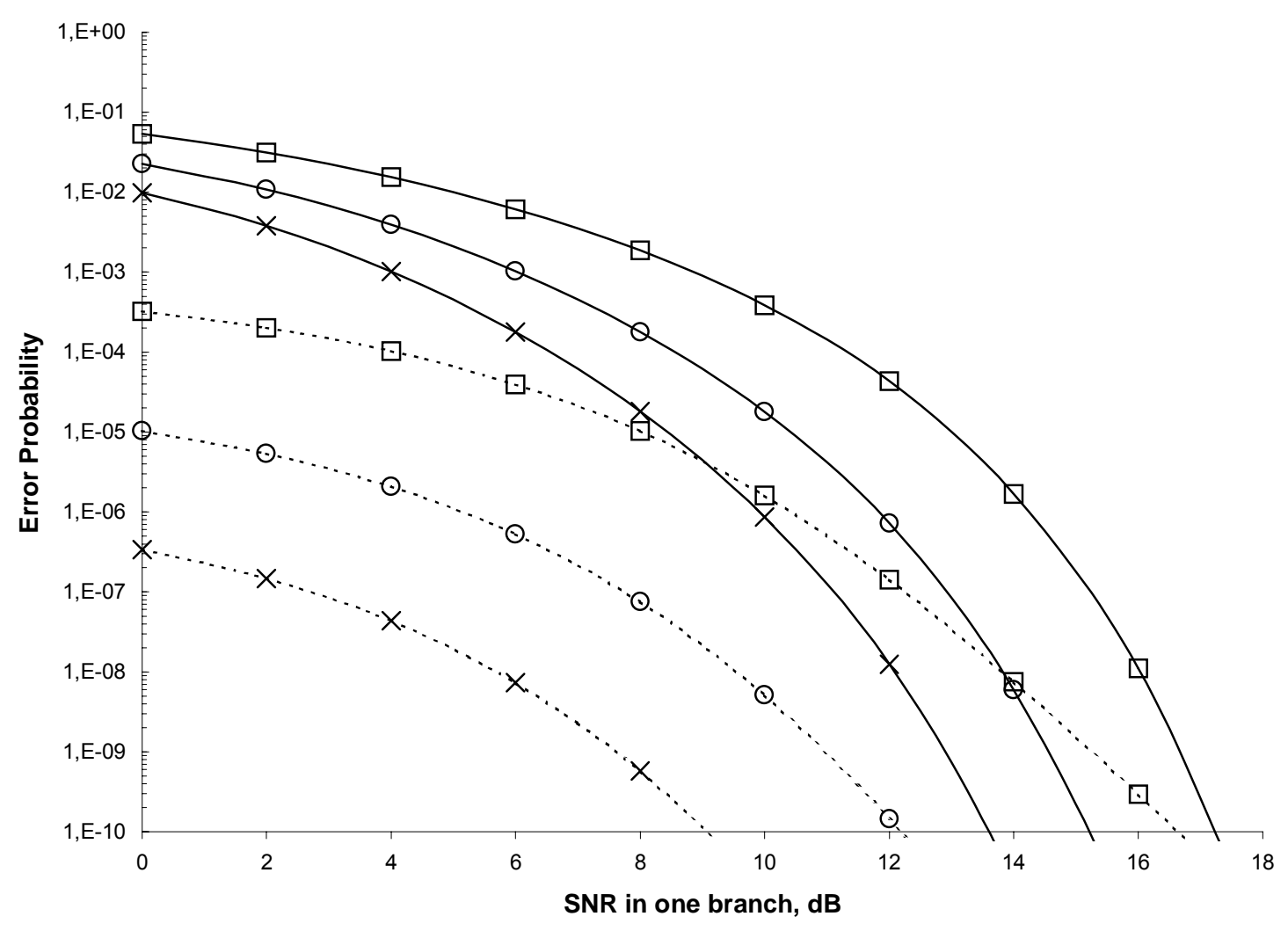


Figure $2 \mathrm{G}$. Karagiannidis and S. Kotsopoulos

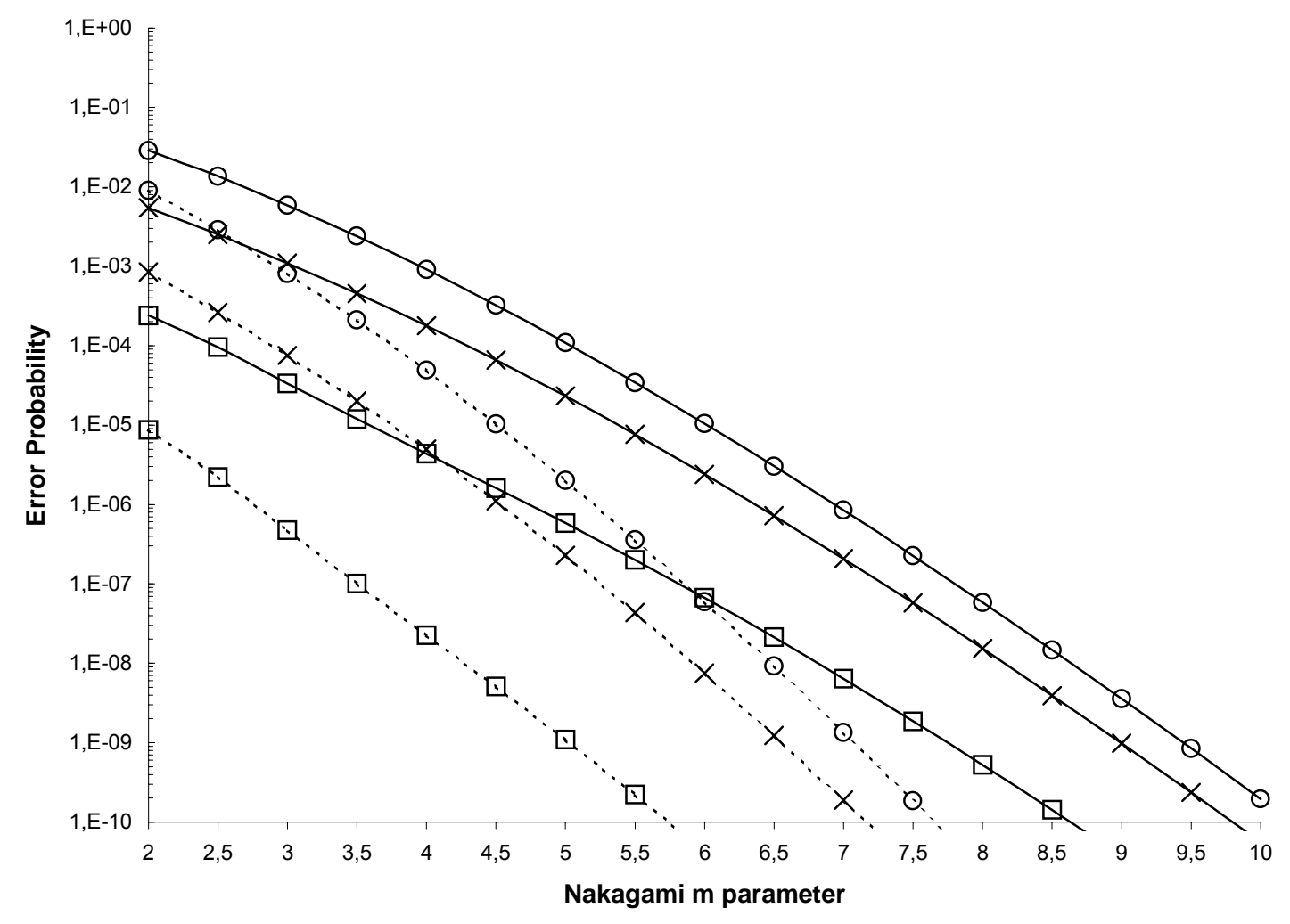

